\title{
Gutiérrez JD, Martínez-Vega RA, Botello H, Ruiz-Herrera FJ, Arenas-López LC, Hernandez-Tellez KD. Environmental and socioeconomic determinants of leptospirosis incidence in Colombia. Cad Saúde Pública 2019; 35(3):e00118417.
}

doi: 10.1590/0102-311XER118417

Where it reads:

Juan David Gutiérrez 1

Ruth Arali Martinez-Vega 2,3

Hector Botello 4,5

Freddy Jesús Ruiz-Herrera 3,5

Laura Carolina Arenas-López 5

Karen Dayana Hernandez-Tellez 5

1 Facultad de Ingeniería, Universidad de Santander, Bucaramanga, Colombia.

2 Escuela de Medicina, Universidad de Santander, Bucaramanga, Colombia.

3 Ultimate Pipe Control, Bucaramanga, Colombia.

4 Facultad de Ciencias Económicas, Universidad Nacional, Bogotá, Colombia.

${ }_{5}$ Escuela de Economía y Administración, Universidad Industrial de Santander, Bucaramanga, Colombia.

It should read:

Juan David Gutiérrez 1

Ruth Arali Martinez-Vega 2

Hector Botello 3,4

Freddy Jesús Ruiz-Herrera 4

Laura Carolina Arenas-López 1

Karen Dayana Hernandez-Tellez 1

${ }^{1}$ Facultad de Ingeniería, Universidad de Santander, Bucaramanga, Colombia.

2 Escuela de Medicina, Universidad de Santander, Bucaramanga, Colombia.

3 Universidad Nacional, Bogotá, Colombia.

${ }_{4}$ Escuela de Economía y Administración, Universidad Industrial de Santander, Bucaramanga, Colombia. 